klärt: so kann Niemand gegen diese Ansicht Etwas einwenden; allein da man die oben genannten Arten als verschiedene gelten lässt: muss man dem graurückigen Fliegenfänger dasselbe Recht widerfahren lassen. Es zeigt sich bei unserm Vogel dieselbe Erscheinung, welche wir bei einigen andern, namentlich bei Sylvia (Curruca) ruficapilla Landbeck und wenn die Beschreibung Deglands richtig ist, bei meiner Ruticilla montana, (Erithacus caïrii Degl.,) wieder finden, nämlich, dass das Männchen die weibliche Zeichnung das ganze Leben hindurch trägt.

Dass die bei Hrn. v. Home yer nistenden graurückigen Fliegenfänger, wie er behauptet, im Hochzeitskleide wirklich frische Federn haben - sie rühren von einer Wintermauser her - beweisen zwei Vögel, welche ich seiner Freundschaft verdanke; sie sind so ächte Muscicapa muscipeta, wie man sie nur wünschen kann, ohne eine Spur von Schwarz in der Zeichnung.

Ich lasse Jedermann seine Ansicht über diese Musc, muscipeta unangefochten; für mich aber sind die vielen und genauen, an Ort und Stelle gemachten Beobachtungen des Hrn. v. Homeyer entscheidend. Ueber Muscicapa parva künftig.

\title{
Ueber die nordamerikanische Elster, (Pica hudsonica Bonap.)
}

\section{Von}

\section{Max Prinz von Wied.}

Es ist bekannt, dass die nördlichen Zonen der verschiedenen Welttheile unserer Erde grosse Uebereinstimmung in ihren thierischen Formen zeigen. Mehrere Thierarten sind über die arctische Zone von Nord-Europa, Asien und Amerika gleichartig verbreitet, andere zeigen nur kleine Abweichungen, sind einander aber höchst nahe verwandt. Bei der früheren oberflächlichen Behandlung der Zoologie nahm man alle jene sich zum Theil sehr ähnliche Thierarten für ein und dieselben Species, indem man sie nicht einmal als verschiedene Abarten oder Raçen betrachtete. In der neueren Zeit ist man nun durch genauere Beobachtung so weit gelangt, unter jenen für identisch angenommenen Arten constante Verschiedenheiten aufzufinden und sie werdeu daher jetzt zum Theil specifisch getrennt. Noch herrschen uber einige derselben Zweifel, welche aber nun sämmtlich in kurzer Zeit aufgeklärt sein werden. Man verfält nur zu leicht aus einem Extrem in das andere, und so ist es auch wohl der Fall, dass man Thiere specifisch trennt, welche sich nur durch unbedeutende Züge unterscheiden, welche 
nur als Abart aufzuführen sein würden. $\mathbf{Z u}$ den eben genannten, in Europa und in Nord-Amerika sich sehr ähnlichen und nahe verwandten Thieren gehört nun vorzüglich auch die Elster, (Pica hudsonica Bonap.,) und es verlohnt sich daher wohl der Mühe diesen Vogel genau erkennen zu lernen, um zu beurtheilen, ob derselbe nur Abart oder wirklich verschiedene Species von der europäischen Elster sei. Wir wollen versuchen, in den nachfolgenden Zeilen eine Beschreibung der amerikanischen Elster nach dem Leben zu geben.

Bei der neueren Verfahrungsweise in der Ornithologie, wo man beinahe alle Species, ganz unbedeutender Abweichungen zufolge, zu Gattungen erhebt, wo man daher ohne alle hinlängliche Charakterzüge die Genera bildet, wo z. B. ein kleiner Federbusch, oder die Färbung des Gefieders zu generischer Trennung berechtigt, da ist es nicht einem jeden Freunde dieser schönen Wissenschaft gegeben, sich an der Schöpfung unzähliger, das Gedächtniss überfüllender barbarischer Benennungen zu betheiligen, man wird aber immer noch hinlänglich Arbeit in diesem Felde finden, wenu man sich nur der gründlichen Beobachtung und Beschreibung der Natur befleissigt, möge dann die weitere Verarbeitung des Materials andern Forschern überlassen bleiben.

Pica hudsonica Bonap. Die nordamerikanische Elster.

(The common Magpie, Audub. Birds IV. p. 99. tab. 227.)

Beschreibung eines weiblichen Vogels, am 9. October bei Fort Union, am oberen Missouri-Laufe, erlegt: Schnabel scheinbar etwas dicker, d. h. höher, als an der europäischen Elster, doch im Allgemeinen von gleicher Gestalt, kürzer als der Kopf, ziemlich hochgewölbt; Nasenlöcher mit vorstrebend anliegenden Borsten bedeckt; Kuppe des Oberkiefers ein wenig über die des untern herabtretend; Kinnwinkel beinahe halb Schnabellänge, rundlich zugespitzt, befiedert, die Federn haben Borstspitzen; Zunge flach mit einer abgesetzt vortretenden Hornspitze, welche regelmässig in zwei gleiche, zugespitzte Hornschenkel getheilt ist; Augenlider an der Aussenseite mit zerstreuten kleinen Pinselfederchen besetzt; Kopf dick und dicht befiedert; Hals kurz; die fünfte Schwungfeder ist die längste, die vierte und sechste sind beinahe nicht kürzer, die erste Feder ganz klein, etwas sichelförmig gekrümmt, dabei sehr schmal lanzettförmig; fünf vordere Schwungfedern mit einem Ausschnitte an der Hinterfahne; Schwanz zwölffederig, lang und stark abgestuft, die beiden mittleren Federn zwei Zell länger, als die nachfolgenden, alsdann fallen sie paarweise, (d. h. an beiden Seiten gezählt,) ziemlich mit 1-1 $1 \frac{1}{2}$ Zoll ab; Beine stark; Fersenrücken mit 6 feinen Tafeln belegt, von welchen die unterste 
schmal ist, die siebente liegt unter den Schenkelfedern verborgen und man bemerkt nur ihren Unterrand; innere Vorderzehe kürzer, als die äusserste, Mittelzehe länger, als beide, Hinterzehe stark, ihr Nagel ist der grösste; Fersensohle scharf kantig zusammengedrückt, mit einer langen Tafel gestiefelt.

Färbung: Schnabel, Beine und Klauen schwarz; Iris im Auge graubraun, allein nach Aussen mit einem bläulich-grauen Ringe umgeben, der der europäischen Elster fehlt, wo überhaupt die Iris ganz dunkel ist; Kopf, Hals, Rücken und Brust kohlschwarz, doch schillert diese Farbe am Ende des Rückens und an den Seiten desselben schön bouteillengrün; an der Kehle haben die an ihrer Spitze schwarzen Federn schneeweisse Wurzeln, ein Zug der der europäischen Elster fehlt; Scapular- und alle oberen Seitenfedern des Rückens, sowie ein Querstreif über den Unterrücken hinweg sind schön rein weiss, ebenso der Bauch, die Seiten und die Aftergegend; Schenkel und Steiss sind schwarz; innere Flügeldeckfedern schwarz mit stahlblauem Glanze; zehn vordere Schwungfedern weiss an ihrer Wurzel; ihre Vorderfahne und der Hinterrand an der Hinterfahne, sowie die Spitze sind schwarz, die Mitte der Hinterfahne weiss, der Rand der Vorderfahne hat grünen Schiller, ebenso die hinteren Schwungfederfahnen zum Theil; die erste dieser schwarzen Fahnen hat einen kleinen runden, weissen Fleck in ihrer Mitte; Flügeldeckfedern ebenso mit stahlblauem und kupfergrünem Schiller; Schwanzfedern sämmtlich an ihrer āusseren Fahne, und die Mittelfedern durchaus prächtig kupfergrün schillernd, aber ein Paar Zoll von der Spitze befindet sich eine violettroth schillernde, oben und unten gelblich-kupfergrün eingefasste Stelle, die Spitze ist wieder grün; innere Fahne der äusseren Schwanzfedern schwarz ohne Glanz.

Ausmessung. *) Weibl, amerikanische Elster.

Länge . . . . . . . $18^{\prime \prime} 8^{\prime \prime \prime}$,

Breite

Länge des Schnabels

Breite des Schnabels

Höhe des Schnabels.

Länge des Flügels .

Länge des Schwanzes

Höhe der Ferse.

Länge der Mittelzehe . . . - 11"'.

Länge der äusseren Zehe
Weibl. europ. Elster.

$$
\begin{array}{cc}
16^{\prime \prime} & 1^{1} / 2^{\prime \prime \prime} . \\
- & - \\
1^{\prime \prime} & 4 / 5^{\prime \prime \prime} . \\
- & 5^{\prime \prime \prime} . \\
- & 5^{\prime \prime \prime} . \\
6^{\prime \prime} & 8^{1} / 2^{\prime \prime \prime} . \\
7^{\prime \prime} & 8^{\prime \prime \prime} . \\
1 " \prime & 82 / 3^{\prime \prime \prime} . \\
- & 10^{1 / 2} / 2^{\prime \prime \prime} \\
- & 6^{7} / 8^{\prime \prime \prime} .
\end{array}
$$

") Ich habe gegen die Ausmessung der amerikanischen Elstern sogleich auch die der europäischen gesetzt. 
Ausmessung. Weibl. amerikanische Elster.

Länge der inneren Zehe . . . $7^{3} / 4^{\prime \prime \prime}$.

$\eta$ der Hinterzehe . . . . $\boldsymbol{7}^{\prime \prime \prime}$.

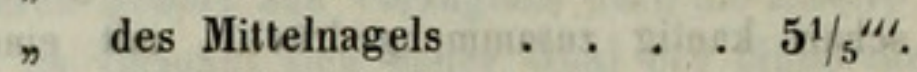

$\eta$ des äusseren Nagels . . . $4^{\prime \prime \prime}$.

7 des inneren Nagels . . . $4^{\prime \prime \prime}$.

" des Hinternagels . . . . $6^{1 / 4}{ }^{\prime \prime \prime}$.
Weibl europ. Elster.

$71 / 2^{\prime \prime \prime}$.

$6^{3} / 4^{\prime \prime \prime}$.

$3^{4} / 5^{\prime \prime \prime}$.

$5^{\prime \prime \prime}$.

Beschreibung einer männlichen Elster, im December bei Fort Union erlegt:

Kopf- und Halsfedern mit etwas blauem Stahlglanze; Seitenfedern des Rückens mit grünem Metallglanze; grosse Flügeldeckfedern und hintere Schwungfedern an ihrer Vorderfahne stahlblau mit kupfergrünem Vorderrande, die Hinterfahne ist schwärzlich matt, ohne Glanz; Scapularfedern breit schneeweiss, ebenso der Unterrücken; obere Schwanzdeckfedern schwarz mit blauem Stahlglanze; an der ganzen Kehle, Unterhals und Oberbrust hat jede Feder vor ihrer Wurzel einen starken weissen Fleck, dagegen sind die Spitzen schwarz und decken die weisse Farbe zu, so dass sie beinahe nicht bemerkt wird, nur an der Kehle ist dieselbe ein wenig sichtbar; innere Flügeldeckfedern schwarz oder doch schwärzlich, hier und da am Rande ein wenig bläulich schillernd. Uebrigens ist die Zeichnung wie beim weiblichen Vogel.

Ausmessung des männl. amerikanisch. Vogels.

Länge. . . . . . . . $19^{\prime \prime} 1^{\prime \prime \prime}$.

Breite

Länge des Schnabels

" des Flügels . . . . $8^{\prime \prime} 5^{1 / 2}{ }^{\prime \prime \prime}$.

" des Schwanzes . . . 11" 11" .

Höhe der Ferse etwa . . . $1^{\prime \prime} 10^{1 / 2}{ }^{\prime \prime \prime}$.

Länge der Mittelzehe . . $-11^{\prime \prime \prime}$.

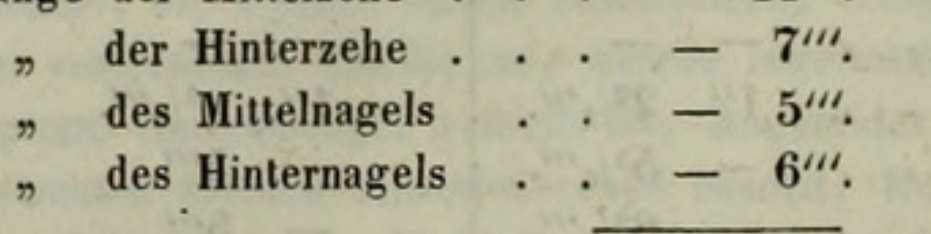

Männl. europ. Vogel.

$17^{\prime \prime} 2^{\prime \prime \prime}$.

$\begin{array}{ll}- & - \\ 1^{\prime \prime} & 2^{\prime \prime \prime} \\ 7^{\prime \prime} & 1^{\prime \prime \prime} \\ 9^{\prime \prime} & 5^{\prime \prime \prime} \\ 1^{\prime \prime} & 9^{\prime \prime \prime} \\ 1^{\prime \prime} & - \\ - & 6^{1} / 3^{\prime \prime \prime} \\ - & 4^{\prime \prime \prime} \\ - & 5^{\prime \prime \prime}\end{array}$

Eine dritte amerikanische Elster, männlichen Geschlechts, am 17. Januar erhalten:

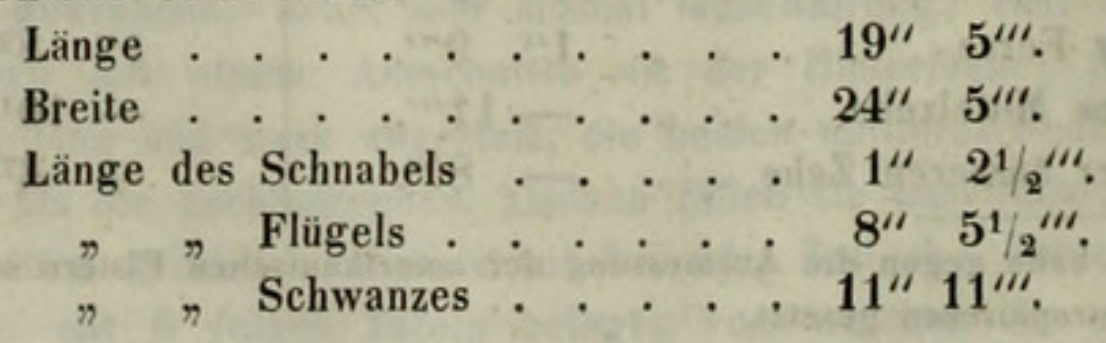




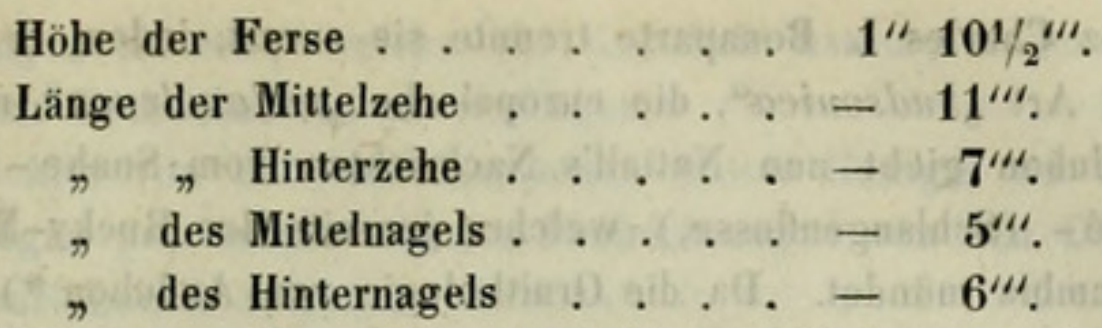

Im Uebrigen war dieser Vogel nicht verschieden von den beiden früher beschriebenen, die Kehlfedern weiss gefleckt, doch in ruhiger Lage unbemerkt.

Die nordamerikanische Elster wurde noch von Richardson und Swainson für identisch mit der europäischen gehalten. In der „Fauna boreali americana" \#) liest man Nachfolgendes von ihr: Sie sei häufig in den innern Prairies, aber sehr selten in den atlantischen Staaten von Amerika. An der Hudsonsbay ist sie einzeln vorgekommen. Nur verirrte Individuen sollen, nach Thomas Say's Zeugniss, östlich vom Missisippi oder am See Winipik vorkommen. Nach diesem Beobachter überwintert die Elster am Missouri und zieht gegen Ende März nördlich. Selbst im Winter sollen sie die Ufer des Saskatschawan nicht verlassen, daselbst im Sommer aber weit häufiger sein. Vergleiche man die Eier der amerikanischen Elster mit der europäischen, so seien die ersteren länger und schmäler, die Farbe dieselbe, allein die Flecken seien bei dem amerikanischen Vogel grösser.

Audubon wiederholt Alles, was Richardson von unserem Vogel sagte, setzte aber noch hinzu, man habe keine von diesen Elstern weiter nördlich gesehen, als von den Quellen des Red River in Louisiana, wo sie Lieutenant Pike zur Zeit seiner Reise in Menge fand. Schon Wilson theilte Pike's Nachrichten mit und Audubon wiederholt sie. Die Elstern setzten sich seinen Pferden auf den Rücken, wo dieselben wund oder gedrückt waren und verursachten ibnen Schmerzen und Unruhe. Sie waren so dreist, sich auf die Arme seiner Leute zu setzen und diesen das Fleisch aus der Hand zu nehmen. Nach Audubon waren es zuerst die Reisenden Lewis und Clarke, welche diesen Vogel in die amerikanische Ornithologie einführten. Sie fanden ihn zuerst am Great Bend, (dem grossen Bogen des Missouri **), obgleich man ihn schon an der Hudsons-Bay beobachtet hatte.

Die Ansichten über die Identität der amerikanischen mit der europäischen Elster sind nach Audubon verschieden. Nuttall hielt beide Vögel für ein und dieselbe Species, Oberst Sabine war anderer Ansicht

*) Vol. II. Birds p. 292. Swainson sagt, er habe zwischen der amerikanischen und europäischen Elster durchaus keinen Unterschied auffinden können.

**) S. die Beschreibung meiner Reise nach Nord-Amerika, Bd. . ., p. ... 
und Prinz Charles L. Bonaparte trennte sie zuerst, indem er die amerikanische Art "hudsonica“, die europäische „melanoleuca" nannte.

Audubon giebt nun Nuttall's Nachrichten vom Snake-River oder Shoshoné - (Schlangenflusse,) welcher jenseits der Rocky-Mountains in den Columbia mündet. Da die Ornithologie von Audubon *) wohl nicht in Jedermanns Händen ist, so wollen wir nachstehend Nuttall's dort angeführte Beobachtungen hier in der Uebersetzung folgen lassen. $\mathrm{Er}$ sagt: „Am 15. Juli, als wir an den Ufern des Shoshoné oder SnakeRiver ankamen, trafen wir zum ersten Mal auf unserer Reise die gemeine Elster, welche sich daselbst gewöhnlich in Gesellschaft des Raben, aber nicht der Krähen fand. Die jungen Vögel, die ihr Futter in der Nähe unseres Lagers suchten, waren so zutraulich und gierig, dass sie von den indianischen Knaben leicht gefangen und bald gezähmt wurden. Die alten Vögel waren ziemlich schüchtern, allein die jungen hüpften nahe umher und liessen ihre Stimmen hören, indem sie, nach Art der Geier, an den von uns weggeworfenen Abfällen von altem Fleische zerrten. In soweit unterschieden sie sich von den ausgestossenen und verfolgten europäischen Elstern, dass wenigstens die Jungen die menschliche Gesellschaft suchten und nicht die geringste Furcht vor uns zeigten, da wir sie mit Nahrung versorgten. Wurden sie für einen Augenblick verjagt, so kehrten sie sogleich zurück und ihre monotonen und gierigen Töne wurden den ganzen Tag von uns vernommen. Die Trockenheit der Jahreszeit und die dadurch verursachte Seltenheit der Insecten und kleinen Vögel zwangen sie ohne Zweifel zu dieser ungewöhnlichen Familiarität mit so zweideutigen Freunden, als den Menschen. An den Ufern der Flüsse im centralen Tafellande der Rocky Mountains fanden wir oft die alten Nester dieser Elstern, gewöhnlich in dichten niederen Büschen, in gewöhnlicher Art von oben bedeckt und oben aus verworrenen Zweigstücken gebaut. In den grossen Waldungen am unteren Columbia sahen wir die Elster beinahe nie, ebenso wenig am La Platte und am Missouri, da sie in allen jenen Gegenden nur gelegentliche Besucher sind. In der Nähe von Monterey in Ober-Californien sind sie nicht selten. Ihr gewöhnlicher Ruf ist "päh! päh!“ und ein leises Gezwitscher, wenn sie zusammenkommen. Ich sah eines Tages einen kleinen Flug von jungen Elstern und hörte eine derselben die abwechselnde Stimme des Katzenvogels nachahmen, als sie auf einem Zweige am Wasser sass, ohne Zweifel, um andere Vögel anzulocken und zur Beute zu machen. Zu einer anderen Zeit bemerkte ich einen

*) Audubon, Birds of America, 8vo, 7 Bände mit 500 illum. Kupfern. 
Flug von jungen Elstern, welche die Kühnheit hatten, andere Vögel und selbst den Taubenhabicht zu verfolgen."

Um das hier von der amerikanischen Elster Gesagte zu vervollständigen, mögen nun unsere eigenen Beobachtungen von den Ufern des oberen Missouri-Laufes folgen.

Die Elster kommt am unteren Missouri nicht vor, und man muss weit aufwärts reisen, bevor man sie beobachtet; ob sie sich aber zuweilen dahin verstreicht, ist mir nicht bekannt. Am oberen Missouri und am Yellow-Stone-Flusse ist sie häufig, am letzteren besonders, wie man sagte, soll sie aber im Herbste und Winter häufiger sein, als im Sommer. Den Missouri aufwärts reisend beobachteten wir sie am 9. Juni zuerst bei Caution Island von Lewis und Clarke, nicht weit unterhalb der Arikkara-Dörfer. Nach Nuttall soll sie nicht bis zu den Mandan-Dörfern hinabgehen und nur in den Rocky-Mountains nisten, was also beides unrichtig ist.

In den Weidengebüschen am Missouri bei Fort Union und von da aufwärts, war die Elster überall nicht selten, oft sehr zahlreich, denn Niemand stellt ihnen nach. Sie ist am oberen Missouri ein Standvogel, indem viele daselbst nisten und auch den Winter zubringen. Sie bleibt im Winter hier, zieht sich aber der Kälte wegen mehr in die Gebüsche, wie man sagte, doch kommt sie in beschützter Lage den Wohnungen alsdann nahe.

Auf der Reise von Fort Union aufwärts, fanden wir in der ersten Hälfte des Monats Juli mehrere Nester dieser Vögel mit schon beinahe erwachsenen Jungen, deren wir aber merkwürdiger Weise nie mehr als zwei Stück fanden, welche gewöhnlich noch nicht recht fliegen konnten und dicht aneinander gedrängt auf der Spitze des dichten, 8-10 Fuss hohen Dornstrauches sassen, in welchem weiter unten, in dichter Verflechtung der Zweige, das grosse, aus dürren Reisern und anderen Materialien zusammengefilzte Nest stand. Die Eier des Vogels haben wir nie gefunden, da es schon spät im Sommer war und grosse Trockenheit herrschte, wo die Vögel nicht viel Nahrung finden. Sie nisten zuweilen auch auf hohe Pappeln, wie bei uns, doch gewöhnlich in einem niederen Strauche. Die alten Vögel sahen wir von den Nestern ab und zu in die Prairie fliegen. Später sahen wir die Elstern am Rande der Prairie, wo dieselbe an die Gebüsche grenzte.

Im Herbste und Winter sind diese Vögel sehr zutraulich. Schon im October sassen sie zu Fort Union auf den Dächern der Gebäude. In der strengen Zeit des Winters, der letzten Hälfte des Decembers und dem Anfange des Januars, waren sie zu Fort Clarke sehr selten, 
sie hatten ohne Zweifel die Gebüsche und die Waldungen gesucht. Nur Kolkraben und Schneeammern, sowie die Linaria sah man alsdann in der offenen Prairie. Das Gefieder der Elstern war in jener Zeit sehr schön und vollständig, der Schwanz nicht abgenutzt.

Die Bemerkung von der geringen Schüchternheit dieses Vogels in wenig besuchten Gegenden, haben auch wir zu machen Gelegenheit gehabt. Während das mit Menschen besetzte Boot den Missouri hinabtrieb, kamen sie uns sehr nahe, umflatterten rufend das Schiff und setzten sich neben uns auf dessen Spitze und Bord, weil wir an einem colossalen, am Vordertheile befestigten Hirschgeweihe die Gewohnheit befolgten, das frisch erlegte Wildpret der Busonten und Hirsche aufzuhängen, die unsere tägliche Nahrung ausmachten. Am Ufer setzten sie sich in unserer Nähe und am Bivouakfeuer auf einen Zweig und gaben, wie unsere Häher, allerhand leise Stimmen von sich, gerade wie dieses Nuttall ganz richtig beschreibt. Sie nehmen alsdann allerhand Stellungen an und wippen mit dem Schwanze.

Ihre Stimme ist gänzlich verschieden von der der europäischen Elster. Sie besteht aus ein Paar hellen Tönen, wie ,giäck! giäck!“ und die leiseren Töne bilden ihre Privatunterhaltung. Man erzählt überall, dass sie das kranke Vieh anfallen und dass sie ein solches Thier tödten und verzehren.

Die Ojibuas nennen die Elster „Akuan-Guisch.“

Um nun schliesslich die Unterschiede noch zusammenzustellen, wodurch sich die amerikanische Elster von der europäischen unterscheidet, so sind dieselben etwa die nachfolgenden :

1) Die amerikanische Elster ist grösser;

2) die Iris in ihrem Auge hat einen graublauen Aussenring, während das Auge der europäischen ganz dunkel ist;

3) der Schnabel des amerikanischen Vogels ist im Verhältniss grösser und dicker, als der des europäischen.

4) Der Unterhals des amerikanischen Vogels hat an der Wurzel weissgefleckte Federn, da sie am europäischen gänzlich schwarz sind.

5) Die Verhältnisse des Körpers sind nach den oben angegebenen Ausmessungen zu vergleichen.

6) Die Stimme beider Vögel ist gänzlich verschieden.

7) Der amerikanische Vogel bringt nur zwei Junge aus.

8) Seine Eier sind anders gebildet und auch ein wenig verschieden gefärbt, 

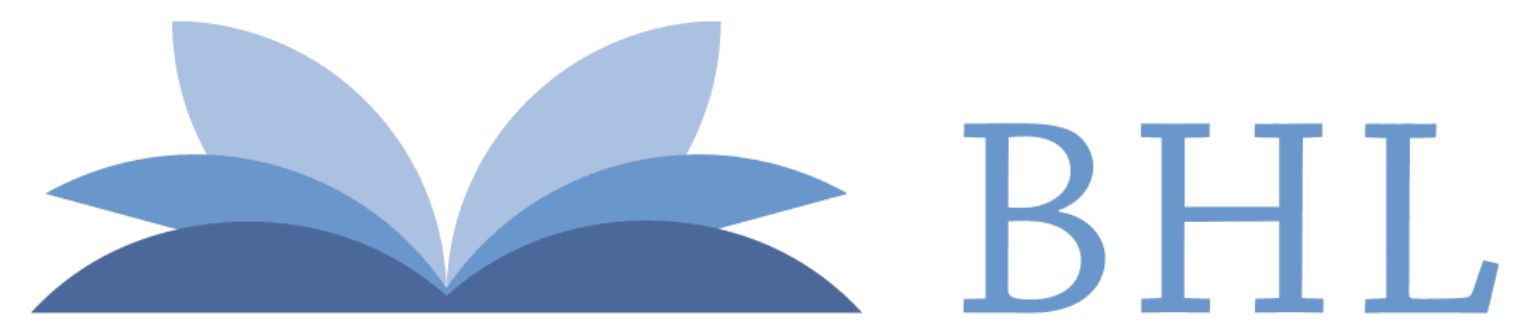

\section{Biodiversity Heritage Library}

1856. "Ueber die nordamerikanische Elster, (Pica hudsonica Bonap.)." Journal fu

r Ornithologie 4, 197-204. https://doi.org/10.1007/bf02002055.

View This Item Online: $\underline{\text { https://www.biodiversitylibrary.org/item/49380 }}$

DOI: $\underline{\text { https://doi.org/10.1007/bf02002055 }}$

Permalink: https://www.biodiversitylibrary.org/partpdf/141237

\section{Holding Institution}

Natural History Museum Library, London

\section{Sponsored by}

Natural History Museum Library, London

\section{Copyright \& Reuse}

Copyright Status: Public domain. The BHL considers that this work is no longer under copyright protection.

This document was created from content at the Biodiversity Heritage Library, the world's largest open access digital library for biodiversity literature and archives. Visit BHL at https://www.biodiversitylibrary.org. 